5 Howard R, Thanos K, Power K, Mahoney A. From childhood trauma to self-harm: an investigation of theoretical pathways among female prisoners. Clin Psychol Psychother 2017; 24(4): 942-51.

6 Hughes K, Bellis M, Hardcastle K, Sethi D, Butchart A, Mikton C, et al. The effect of multiple adverse childhood experiences on health: a systematic review and meta-analysis. Lancet Public Health 2017; 2(8): e356-66.

7 Tully J, Cappai A, Lally J, Fotiadou M. Follow-up study of 6.5 years of admissions to a UK female medium secure forensic psychiatry unit. BJPsych Bulletin 2019; 43(2): 54-7.

8 Covington S, Russo R. Healing Trauma: A Brief Intervention for Women, 1st ed. Hazelden Publishing, 2011.

9 Covington S. Becoming Trauma Informed: A Training for Correctional Professionals, 1st ed. Center for Gender \& Justice, 2012.

10 World Health Organization (WHO). International Statistical Classification of Diseases and Related Health Problems 10th Revision. WHO, 2016 (https://icd.who.int/browse10/2016/en).

11 UK Government. Mental Health Act 1983. HMSO, 1983 (https://www. legislation.gov.uk/ukpga/1983/20/contents).

12 Felitti VJ, Anda R F, Nordenberg D, Williamson D, Spitz A, Edwards V, et al. Relationship of childhood abuse and household dysfunction to many of the leading causes of death in adults. The Adverse
Childhood Experiences (ACE) Study. Am J Prev Med 1998; 14(4): 245-58.

13 Ribeiro RB, Tully J, Fotiadou M. Clinical characteristics and outcomes on discharge of women admitted to a medium secure unit over a 4-year period. Int J Law Psychiatry 2015; 39: 83-9.

14 Baker A, Wright K, Hansen E. A qualitative study exploring female patients' experiences of self-harm in a medium secure unit. J Psychiatr Ment Health Nurs 2012; 20(9): 821-9.

15 Muskett C. Trauma-informed care in inpatient mental health settings: a review of the literature. Int J Ment Health Nurs 2014; 23(1): 51-9.

16 Cleare S, Weatherall K, Clark A, Ryan C, Kitley O, Smith M, et al. Adverse childhood experiences and hospital-treated self-harm. Int Environ Res Public Health 2018; 15(6): 1235

17 Hare Duke L, Furtado V, Guo B, Völlm B. Long-stay in forensic-psychiatric care in the UK. Soc Psychiatry Psychiatr Epidemiol 2018; 53(3): 313-21.

18 Klonsky ED, Glenn CR. Assessing the functions of non-suicidal self-injury: psychometric properties of the Inventory of Statements About Self-injury (ISAS). J Psychopathol Behav Assess 2009; 31(3): 215-9.

\title{
AGAINST THE STREAM
}

\section{Psychiatrists should investigate their patients less}

\author{
Matthew Butler, ${ }^{1,2} \odot$ Fraser Scott, ${ }^{2}$ Biba Stanton, ${ }^{2,3}$ Jonathan Rogers ${ }^{4} \odot$
}

BJPsych Bulletin (2022) 46, 152-156, doi:10.1192/bjb.2021.125

${ }^{1}$ King's College London, UK; ${ }^{2}$ South London and Maudsley NHS Foundation Trust, London, UK; ${ }^{3}$ King's College Hospital, London, UK; ${ }^{4}$ University College London, UK

Correspondence to Matthew Butler (matthew.butler@kcl.ac.uk)

First received 11 Jun 2021, final revision 1 Oct 2021, accepted 10 Nov 2021

(c) The Author(s), 2021. Published by Cambridge University Press on behalf of the Royal College of Psychiatrists. This is an Open Access article, distributed under the terms of the Creative Commons Attribution licence (https:// creativecommons.org/licenses/by/4. $0 /$ ), which permits unrestricted re-use, distribution, and reproduction in any medium, provided the original work is properly cited.
Summary Psychiatrists often order investigations such as blood tests, neuroimaging and electroencephalograms for their patients. Rationales include ruling out 'organic' causes of psychiatric presentations, providing baseline parameters before starting psychotropic medications, and screening for general cardiometabolic health. Hospital protocols often recommend an extensive panel of blood tests on admission to a psychiatric ward. In this Against the Stream article, we argue that many of these investigations are at best useless and at worst harmful: the yield of positive findings that change clinical management is extremely low; special investigations are a poor substitute for a targeted history and examination; and incidental findings may cause anxiety and further unwarranted investigation. Cognitive and cultural reasons why over-investigation continues are discussed. We conclude by encouraging a more targeted approach guided by a thorough bedside clinical assessment.

Keywords Service users; in-patient treatment; imaging; ethics; cost-effectiveness.
The Royal College of Psychiatrists' Standards for Inpatient Mental Health Services require a 'comprehensive physical review' to be initiated within four hours of admission to a psychiatric inpatient unit, which is to be completed within one week. ${ }^{1}$ In practice, this means that inpatients are often subjected to a battery of admission blood tests that are directed without regard for the presentation. Routine blood tests on admission remain a requirement in local trust policies, ${ }^{2}$ and clinical evaluations indicate that the majority of patients receive them. ${ }^{3}$ In addition, selected psychiatric patients may have further investigations, such as a magnetic resonance imaging (MRI) of the brain for those with a first psychotic episode, or an electroencephalogram (EEG) to rule out seizures. 
In this article, we consider some of the reasons why investigations might be requested by psychiatrists and why they are often inappropriate, and suggest an alternative approach.

\section{The case for investigating}

There are several (non-exhaustive) reasons why investigations might be requested for psychiatric patients:

- to screen for contributing physical factors in the acute presentation, including concern that rare but serious causes are not missed

- to provide baseline levels that may be relevant when starting particular psychotropic medications

- to screen for a range of chronic physical illnesses that might otherwise be missed because psychiatric patients may not present as often to healthcare professionals or are treated differently when they do

- to screen for health problems that are more common in those with severe mental illness, such as diabetes and liver disease.

Here, a distinction should be made between screening tests and diagnostic tests. Screening tests are those performed at population or subpopulation level to detect presymptomatic disease with the aim of early intervention. An example of this may be routine prediabetes screening in a patient on chronic antipsychotic treatment. Screening tests have been used in medicine since the middle of the 20th century (an early example being the successful screening and treatment of syphilis) and have undoubtedly improved diagnosis rates for medical and psychiatric patients. ${ }^{4}$ Despite this, there are a number of strict criteria that a screening test must meet before it is acceptable for use, including prior implementation of cost-effective primary prevention, a validated test, agreement on further investigation, good evidence for intervention at the presymptomatic phase, and evidence that benefits outweigh harms.

Screening tests are distinct from diagnostic tests, which aim to accurately diagnose a condition for which there is clinical suspicion. An example of this may be positive antineuronal antibodies in the cerebrospinal fluid of a patient with clinically suspected autoimmune psychosis.

Although there are undoubtedly many cases where screening and diagnostic tests are entirely justified, we believe that investigations are often inappropriate because they have a low yield, poor sensitivity and specificity, limited interpretability without clinical correlation, and the potential to cause serious harm.

\section{The case for not investigating}

\section{Tests may miss what they are looking for or not alter management}

For an investigation to be useful, it should have high sensitivity (the ability to correctly identify patients with a given disease) and specificity (the ability to correctly identify patients without said disease). Distinct but related clinically useful concepts are those of positive predictive value (the probability that someone with a positive test has the disease) and negative predictive value (the probability that someone with a negative test does not have the disease). It is of note that even when tests do have high reported sensitivity and specificity, there may be additional complexities in realworld settings. This is in part because when developing tests, figures of diagnostic accuracy are calculated in a specific context, which is often obviously symptomatic individuals compared with obviously healthy controls.

Many diagnostic investigations used by psychiatrists fail to meet these criteria, and many have such a low probability of a clinically relevant result that it is hard to justify their routine clinical use. Systematic reviews have found that routine blood tests rarely alter the management of patients due for admission to psychiatric hospital, even when abnormal results are found. ${ }^{6,7}$ In one of the included studies of 500 patients, only one individual had an abnormal result that mandated urgent medical intervention. Notably, this patient was obviously symptomatic. ${ }^{8}$

Imaging and physiological investigations may not fare better. EEGs show interictal epileptiform discharges (IEDs) in only $29-55 \%$ of people with epilepsy, ${ }^{9}$ so it is inappropriate as a rule-out test for complex partial seizures presenting with psychotic symptoms. In contrast, EEG abnormalities (broader than just IEDs) occur in 19\% of those treated with antipsychotics, so an abnormal EEG is not specific to a neurological cause of psychiatric symptoms. ${ }^{10}$ Similarly, one study found that $6 \%$ of healthy controls have at least one abnormality on an MRI of the brain, so the presence of an abnormality per se has a poor specificity for determining a neurological cause for psychiatric symptoms. This same research also concluded that none of 349 individuals with first-episode psychosis had MRI findings that necessitated a change in clinical management. ${ }^{11}$

\section{Investigations are no substitute for history taking and physical examination}

Psychiatric patients invariably present in complex and myriad ways. In a minority, there is a recognisable physical cause for the psychiatric symptoms. For a physical illness to lead to psychiatric symptoms, there must either be a pathophysiological process (e.g. neoplastic, endocrine, autoimmune, metabolic, epileptiform, infective, neurodegenerative or metabolic) that is affecting the central nervous system and/or psychosocial mechanisms resulting from the physical illness. In each of these cases, it is very unlikely that a patient would present as entirely physically asymptomatic to an appropriately curious and observant clinician.

To put it another way, we might be surprised to see such physical illnesses presenting 'occultly', in the sense that a case would be missed after a thorough history and examination but picked up on a routine blood test or other untargeted investigation. Where psychosocial mechanisms predominate, these generally occur via experience of symptoms or via knowledge of a diagnosis, both of which can be elicited through an appropriate history or examination. In the specific case of functional disorders (such as functional neurological disorders), these are diagnosed through eliciting positive clinical signs, rather than being 'diagnoses 
of exclusion' which require investigation to exclude other causes. ${ }^{12}$

Even for tests with extraordinarily high sensitivity and specificity, there are further interpretative difficulties that arise in the absence of clinical suspicion. If a condition is very rare, then an abnormal investigation result may not be as diagnostic as is intuitively suspected. This is known as the 'false-positive paradox', or the 'base rate paradox', and occurs when the prevalence of a condition is lower than the test's false-positive rate (in these cases, the test will give more false positives than true positives). Crucially, the calculation is entirely different in populations who are already suspected to have the disease based on history and examination. In these cases, the pre-test probability (and thus the post-test probability after a positive test) would be much higher, and a resulting abnormal test much more clinically useful.

Take hypothetical blood test A which has a specificity of $99 \%$ for condition $\mathrm{X}$. If condition $\mathrm{X}$ has a population prevalence of $1 \%$ and there are no additional features to increase the clinical suspicion of the condition (e.g. the patient has not been examined), we can assume the pre-test probability is $1 \%$. In this case, an abnormal result from blood test A translates to a chance of having the condition (i.e. post-test probability) of only 50\%. Most physicians, including us authors, would probably overestimate the post-test probability following a positive result on blood test A.

\section{Investigations can be harmful}

Investigations often have attendant risks, which are seldom adequately considered. In some circumstances, taking blood can be painful or uncomfortable (particularly in a distressed, paranoid or disoriented patient), risking injury for patient and clinician. The noise and enclosed environment of an MRI scanner can be frightening for an anxious patient. Over-investigation may also contribute to the development or perpetuation of health anxiety, as it can serve to confirm a patient's fears about a serious undiagnosed illness. ${ }^{13}$ This may be particularly pertinent for individuals with somatoform or hypochondriacal disorders, who psychiatrists may encounter more often than might clinicians in other specialties.

A further important consideration is the impact of false positives. When tests are ordered in the absence of a clinical suspicion or hypothesis, a positive result inevitably returns the question: what next? In many cases, this might be further investigation, leading to a non-negligible potential of harm. Take the case of creatine kinase (CK), which is sometimes recommended as an admission blood test for psychiatric in-patients, ${ }^{2}$ despite being incidentally and benignly raised in many patients, and even being a poor discriminator for neuroleptic malignant syndrome. ${ }^{14}$ When faced with a raised $\mathrm{CK}$ in an asymptomatic patient, most of us feel that we should do something, so patients are frequently subjected to further blood tests and sometimes intravenous fluids for a test that should never have been done. More dramatically, if ten people are treated with endovascular coiling for an incidental unruptured intracranial aneurysm detected on MRI, one will end up disabled or dead within one year, all for a lesion that probably would not have caused any harm. ${ }^{15}$
Readers may be familiar with 'incidentalomas', an all-encompassing term for non-specific lesions seen on medical imaging. Incidentalomas have dubious clinical relevance, but often prompt further investigations and cause anxiety to patients and professionals alike. Relatedly, the phenomenon of non-specific abnormal results also occurs with blood tests. One study of healthy adults receiving a routine battery of blood tests found that over one-third had at least one abnormal result, of which only $7 \%$ were deemed to require a medical review (none of them urgently). ${ }^{16}$

Finally, in the context of limited healthcare funding, expensive investigations divert resources from more worthwhile causes. Discounting the cost of staff time, a simple routine battery of blood tests is likely to cost in the region of $£ 15-20 .{ }^{17}$ The number of psychiatric admissions needed to screen to find a positive serum free thyroxine (FT4) is in the region of 127 , which corresponds to a cost of around $£ 400$ per abnormal result. ${ }^{18}$ MRI brain scans cost in the region of $£ 200$. Cost-wise, there is likely more benefit from a five minute clinical history and examination.

\section{Cultural and psychological factors}

Psychiatry is a medical discipline and there are, without any doubt, physical causes of psychiatric presentations. In addition, patients with severe mental illness are far more likely to die early, in part owing to huge over-representation of physical comorbidities such as cardiovascular disease. Psychiatrists should be attuned to physical health problems in in-patients as much as mental health problems, and we should feel confident in routinely performing thorough physical health histories and examinations.

The historical and ongoing divide between mental and physical healthcare is also likely to be a factor in overinvestigation of psychiatric patients. Psychiatrists do not always have easy access to opinions from other specialties, and it is unusual for a physician to be integrated into psychiatric services. Psychiatrists may utilise unnecessary investigations when they are worried about patients, instead of being able to access a clinical opinion from specialist physicians. If physical and mental health services were better integrated, this could be avoided.

However, the overuse of investigations is a common theme in much of medicine and often functions more as a 'psychological comfort blanket for clinicians' rather than providing any tangible benefit to our patients. ${ }^{19}$ It may also stem from 'addition bias', the human tendency to try to solve challenging problems by adding something in rather than taking something away. ${ }^{20}$ Ordering an investigation may make us feel as though we have addressed the problem we were trying to solve, when in fact we might not have. For instance, if the problem is poor physical health outcomes in people with severe mental illness, admission blood tests are unlikely to be an answer. Focusing on this superficial attempt at joined-up healthcare perhaps distracts from other solutions, such as addressing stigma and reducing inequalities.

Furthermore, the familiar feeling that we must 'do something rather than nothing' may stem from a human tendency towards action, which is also known as 
intervention bias. ${ }^{21}$ Of course, as we have seen, there are scenarios where doing nothing is just as good, or even better, than doing something. The Hippocratic oath reminds us that our primary role as doctors is to first do no harm.

Overuse of investigations in psychiatry may also represent part of a wider risk-averse culture in medicine, which is sometimes referred to as 'defensive medicine'. Defensive medicine is costly to healthcare institutions. ${ }^{22}$ Many doctors, including ourselves and three-quarters of all psychiatrists, admit to defensive medicine to some degree, including the ordering of clinically useless investigations. ${ }^{23}$ The reasons why clinicians may practise defensive medicine are myriad; however, the most commonly cited reason is fear of litigation resulting from malpractice hearings, particularly as the majority of cases of litigation stem from doctors missing diagnoses, rather than actively causing harm through the use of treatments. ${ }^{22}$ There are some (albeit incomplete) data that some aspects of defensive medicine may stem from physicians struggling to tolerate uncertainty in patient diagnosis and care. ${ }^{24}$

\section{Concluding remarks}

We suggest six points to keep in mind when considering an investigation for our patients.

Limitations of tests

(1) Have we examined the patient and taken a history? Without a thorough examination, performing an investigation may not provide any useful information.

(2) Is the disease common enough that this test will be useful? If the disease or condition is rare, there is a high probability of false positives.

(3) How would we need to act if the test were to return an abnormal result? Additional interventions or investigations that may result could be harmful.

Limitations of human psychology:

(4) Am I doing this test to resolve my own anxieties, or will it benefit the patient? Sometimes doing nothing rather than something is in the patient's best interests.

(5) Can I tolerate the uncertainty of not knowing? Reaching after false certainty is not in anyone's interest.

(6) Is there any chance the test or the results could lead to negative outcomes for the patient? First, do no harm.

We support attempts to integrate the body into mental health and illness. We believe that a good means of helping to achieve this is to ensure that we conduct thorough histories and examinations. This allows us both to request the appropriate investigations and to know how to interpret them once we have the results. This approach is supported by the American Psychiatric Association, which discourages routine laboratory testing, ${ }^{25}$ and by the National Institute for Health and Care Excellence (NICE), which does not recommend neuroimaging in first-episode psychosis. ${ }^{26}$ However, too often doctors are still faced with incidental findings on tests that should never have been requested. Psychiatrists should investigate their patients less and examine them more.

\section{About the authors}

Matthew Butler is a Maudsley BRC Preparatory Clinical Research Training Fellow at the Institute of Psychiatry, Psychology \& Neuroscience, King's College London, and a speciality trainee with South London and Maudsley NHS Foundation Trust, London, UK. Fraser Scott is a speciality trainee with South London and Maudsley NHS Foundation Trust, London, UK. Biba Stanton is a consultant neurologist at King's College Hospital, London, UK and South London and Maudsley NHS Foundation Trust, London, UK. Jonathan Rogers is a Wellcome Trust Clinical Training Fellow at University College London, UK.

\section{Data availability}

Data availability is not applicable to this article as no new data were created or analysed in this study.

\section{Author contributions}

M.B., F.S. and J.R. co-conceptualised the paper. M.B. wrote the first draft, amended subsequent drafts and reviewed the final submission. F.S. and J. R. reviewed and amended subsequent drafts, and reviewed final submission B.S. reviewed and amended drafts and reviewed the final submission.

\section{Funding}

This research received no specific grant from any funding agency, commercial or not-for-profit sectors.

\section{Declaration of interest}

J.R. has received payment from the Alberta Psychiatric Association for a lecture and has held one unpaid advisory meeting with representatives from Promentis Pharmaceuticals regarding drug development.

\section{References}

1 Royal College of Psychiatrists. Standards for Inpatient Mental Health Services (3rd edn). RCPsych, 2019.

2 South London and Maudsley NHS Foundation Trust Clinical Policy Working Group. Physical Healthcare Policy. SLaM, 2019.

3 Hassan S, Byravan S, Al-Zubaidi H. Improving physical healthcare provided to psychiatric inpatients at an acute mental health trust. $B M$ Open Qual 2019; 8: e000537.

4 Morabia A, Zhang FF. History of medical screening: from concepts to action. Postgrad Med J 2004; 80: 463-9.

5 UK National Screening Committee. Criteria for Appraising the Viability, Effectiveness and Appropriateness of a Screening Programme. TSO (The Stationery Office), 2015 (https://www.gov.uk/government/publications/ evidence-review-criteria-national-screening-programmes/criteria-forappraising-the-viability-effectiveness-and-appropriateness-of-a-screeningprogramme).

6 Chennapan K, Mullinax S, Anderson E, Landau MJ, Nordstrom K, Seupaul RA, et al. Medical screening of mental health patients in the emergency department: a systematic review. J Emerg Med 2018; 55 799-812.

7 Conigliaro A, Benabbas $R$, Schnitzer $E$, Janairo M-P, Sinert $R$ Protocolized laboratory screening for the medical clearance of psychiatric patients in the emergency department: a systematic review. Acad Emerg Med 2018; 25: 566-76.

8 Janiak BD, Atteberry S. Medical clearance of the psychiatric patient in the emergency department. J Emerg Med 2012; 43: 866-70.

9 Pillai J, Sperling MR. Interictal EEG and the diagnosis of epilepsy. Epilepsia 2006; 47: 14-22. 
10 Centorrino F, Price BH, Tuttle M, Bahk WM, Hennen J, Albert MJ, et al. EEG abnormalities during treatment with typical and atypical antipsychotics. Am J Psychiatry 2002; 159: 109-15.

11 Falkenberg I, Benetti S, Raffin M, Wuyts P, Pettersson-Yeo W, Dazzan P, et al. Clinical utility of magnetic resonance imaging in first-episode psychosis. Br J Psychiatry 2017; 211: 231-7.

12 Espay AJ, Aybek S, Carson A, Edwards MJ, Goldstein LH, Hallett M, et al. Current concepts in diagnosis and treatment of functional neurological disorders. JAMA Neurol 2018; 75: 1132-41.

13 Howard LM, Wessely S. Reappraising reassurance: the role of investigations. J Psychosom Res 1996; 41: 307-11.

14 Chang CK, Harrison S, Lee W, Taylor D, Stewart R. Ascertaining instances of neuroleptic malignant syndrome in a secondary mental healthcare electronic medical records database: the SLAM BRC case register. Ther Adv Psychopharmacol 2012; 2: 75-83.

15 Al-Shahi Salman R, Whiteley WN, Warlow C. Screening using wholebody magnetic resonance imaging scanning: who wants an incidentaloma? J Med Screen 2007; 14: 2-4

16 Keuss SE, Parker TD, Lane CA, Hoskote C, Shah S, Cash DM, et al. Incidental findings on brain imaging and blood tests: results from the first phase of Insight 46, a prospective observational substudy of the 1946 British birth cohort. BMJ Open 2019; 9(7): e029502.

17 Mughal Z, Narayanan A, Gupta V, Reay-Jones N. Clinical need-directed blood tests: a step in saving the NHS? Ann Clin Biochem 2016; 53: 568-74.

18 Arce-Cordon R, Perez-Rodriguez MM, Baca-Baldomero E, Oquendo MA, Baca-Garcia E. Routine laboratory screening among newly admitted psychiatric patients: is it worthwhile? Psychiatr Serv 2007; 58: $1602-5$

19 Faulkner A, Reidy M, McGowan J. Should we abandon routine blood tests? BMJ 2017; 357: j2091.

20 Adams GS, Converse BA, Hales AH, Klotz LE. People systematically overlook subtractive changes. Nature 2021; 592: 258-61.

21 Foy AJ, Filippone EJ. The case for intervention bias in the practice of medicine. Yale J Biol Med 2013; 86: 271-80.

22 Anderson RE. Billions for defense: the pervasive nature of defensive medicine. Arch Intern Med 1999; 159: 2399-402.

23 Passmore K, Leung W-C. Defensive practice among psychiatrists: a questionnaire survey. Postgrad Med J 2002; 78: 671-3.

24 Alam R, Cheraghi-Sohi S, Panagioti M, Esmail A, Campbell S, Panagopoulou E. Managing diagnostic uncertainty in primary care: a systematic critical review. BMC Fam Pract 2017; 18: 79

25 APA Work Group on Psychiatric Evaluation. The American Psychiatric Association Practice Guidelines for the Psychiatric Evaluation of Adults (3rd edn). American Psychiatric Association, 2016.

26 National Institute for Health and Care Excellence. Structural Neuroimaging in First-Episode Psychosis (Technology Appraisal Guidance TA136). NICE, 2008.

\section{CULTURAL REFLECTIONS}

\section{Evensong: how the medical humanities can strengthen a patient-centred approach to both physical and mental health conditions}

\section{Corinne Rowena Dignan 10}

BJPsych Bulletin (2022) 46, 156-158, doi:10.1192/bjb.2021.3

St Cadoc's Hospital, Newport, UK Correspondence to Dr Corinne Dignan (corinne.dignan@wales.nhs.uk)

First received 16 May 2020, accepted 24 Nov 2020

(c) The Author 2021. Published by Cambridge University Press on behalf of the Royal College of Psychiatrists. This is an Open Access article, distributed under the terms of the Creative Commons Attribution licence (http:// creativecommons.org/licenses/by/4. $0 /$ ), which permits unrestricted re-use distribution, and reproduction in any medium, provided the original work is properly cited.
Summary The medical humanities may offer an antidote to the unconscious depersonalisation of patients into clinical variables and diagnoses at the hands of physicians, cultivating a patient-centred and individual approach to the management of both physical and mental health conditions. The emphasis on the person behind the diagnosis helps physicians to remain motivated and compassionate in the face of increasing social and organisational pressures that threaten this human connection. As a doctor and the relative of a patient with dementia, I reflect on the way in which poetry has helped to translate my experience as a relative into improving my own practice as a doctor. This article includes one of the poems I wrote during my grandmother's illness to aid reflection on the patient perspective I gained during her time in hospital, and also a brief commentary exploring the influence this process has had on the interactions I now have with my patients.

Keywords Education and training; dementia; comorbidity; organic syndromes; stigma and discrimination. 